Check for updates

London, UK

Cite this as: BMJ 2021;372:n567 http://dx.doi.org/10.1136/bmi.n567 Published: 25 February 2021

\section{Covid-19: Pfizer BioNTech vaccine reduced cases by $94 \%$ in Israel, shows peer reviewed study}

\author{
Jacqui Wise
}

The Pfizer BioNTech coronavirus vaccine produces as good results in the "real world" as have been previously documented in randomised trials.

A case control study, which has been peer reviewed and published in the New England Journal of Medicine, compared 596618 people who were newly vaccinated in Israel and matched them to unvaccinated controls. ${ }^{1}$ Two doses of the mRNA vaccine reduced symptomatic cases by $94 \%$, hospitalisation by $87 \%$, and severe covid-19 by $92 \%$, according to the data from the Clalit Institute for Research which is Israel's biggest healthcare provider.

Researchers recorded the outcomes at three periods: days 14 to 20 after the first dose of vaccine, days 21 through 27 after the first dose, and day 7 after the second dose. In Israel, the second dose of vaccine is given on day 21 in line with the trials and the manufacturer's recommendation. The UK is leaving a 12 week gap between doses.

During a mean follow-up of 15 days, 10561 infections were documented of which 5996 were symptomatic covid-19 illness, 369 required hospitalisation, 229 were severe cases, and 41 resulted in death.

After one dose, during days 14 to 20 , the vaccine effectiveness was $57 \%$ for symptomatic covid- $19,74 \%$ for hospitalisation, and $62 \%$ for severe disease. The estimated effectiveness in preventing death from covid- 19 was $72 \%$ in the two to three weeks after the first dose. There was insufficient data to produce an estimate on the reduction in mortality in those who received two doses.

The study took place from 20 December 2020, when Israel's national vaccination drive was launched, to 1 February 2021. It coincided with Israel's third and largest wave of coronavirus infection and illness.

The study also suggests the Pfizer BioNTech vaccine is effective against the B.1.1.7 variant which was first identified in the UK. During the study period, an increasing share of SARS-CoV-2 isolates in Israel-up to $80 \%$-were of this variant. The data, however, provide no information about the effectiveness of the vaccine against the South African variant, B.1.351, as this was estimated to be rare in Israel at the time of the study.

The study authors add that the vaccine effectiveness was similar for adults 70 years of age or older and for younger age groups. However, the study was not designed to systematically assess viral transmission or asymptomatic infections. As Israel does not systematically test asymptomatic people, some asymptomatic cases will have been missed.

Peter English, consultant in communicable disease control and past chair of the BMA's Public Health Medicine Committee, said, "This is more great news, confirming that the vaccine is around 90\% effective at preventing documented infection of any degree of severity from seven days after the second dose. This is consistent with the findings of the phase III trials carried out before the vaccine was marketed."

He added, "Despite it having been published quickly, the quality of the paper is of the highest. It is rigorous and thorough, with appropriate statistical analyses."

Ran Balicer, senior author of the study and director of the Clalit Research Institute, said, "These results show convincingly that this vaccine is highly effective against symptomatic covid-19, one week after the second dose. These results are similar to those reported in the previously published clinical trial, despite the challenges inherent in a mass vaccination setting."

Dagan N, Barda N, Kepten E, etal. BNT162b2 mRNA Covid-19 vaccine in a nationwide mass vaccination setting. N Engl J Med 2021. doi: 10.1056/NEJMoa2101765. pmid: 33626250 